\title{
CUZD1 and Anti-CUZD1 Antibodies as Markers of Cancer and Inflammatory Bowel Diseases
}

\author{
Christos Liaskos, ${ }^{1}$ Eirini I. Rigopoulou, ${ }^{2}$ Timoklia Orfanidou, ${ }^{1}$ \\ Dimitrios P. Bogdanos, ${ }^{1,2,3}$ and Christos N. Papandreou ${ }^{4}$ \\ ${ }^{1}$ Cellular Immunotherapy and Molecular Immunodiagnostics, Institute of Research and Technology Thessaly, \\ 41222 Larissa, Greece \\ ${ }^{2}$ Department of Medicine, Faculty of Medicine, School of Health Sciences, University of Thessaly, Biopolis, \\ 41110 Larissa, Greece \\ ${ }^{3}$ Division of Transplantation Immunology and Mucosal Biology, King's College London School of Medicine, \\ King's College Hospital, Denmark Hill Campus, London SE5 9RS, UK \\ ${ }^{4}$ Department of Medical Oncology, University Hospital of Larissa, Faculty of Medicine, School of Health Sciences, \\ University of Thessaly, Biopolis, 41110 Larissa, Greece
}

Correspondence should be addressed to Dimitrios P. Bogdanos; dimitrios.bogdanos@kcl.ac.uk

Received 16 March 2013; Accepted 29 March 2013

Academic Editor: Yehuda Shoenfeld

Copyright (C) 2013 Christos Liaskos et al. This is an open access article distributed under the Creative Commons Attribution License, which permits unrestricted use, distribution, and reproduction in any medium, provided the original work is properly cited.

CUZD1, the CUB, and zona pellucida-like domains-containing protein 1, is a newly identified antigen of pancreatic autoantibodies $(\mathrm{PAB})$ giving a reticulogranular pattern in patients with inflammatory bowel diseases, and in particular Crohn's disease. The exact mechanisms by which this pancreatic antigen becomes the target of IBD-specific pancreatic autoantibodies are unclear. At the same time, evolving data strongly support a role for CUZD1 in carcinogenesis. Human CUZD1 is mapped at chromosome $10 \mathrm{q} 26.13$ and the loss of this region is a frequent event in various malignant tumours. mRNA overexpression of CUZD1 has been noted in ovarian cancer and serum levels of CUZD1 are elevated in women with ovarian cancer and patients suffering from pancreatic cancer. CUZD1 appears to be one of the relatively few biomarkers that serve as both cancer biomarker and autoantigen of autoantibodies in an autoimmune disease unrelated to cancerous organs. This review discusses the role of CUZD1 in cancer and autoimmunity. We anticipate that a better understanding of the function of CUZD1 will help us to understand how it becomes the focus of an autoimmune attack specifically targeting the intestine and its enigmatic role in carcinogenesis.

\section{Introduction}

In recent years, the identification and validation of novel biomarkers has become the focus of intense research in both the laboratory and clinic. Biomarkers now have several applications and their role has been extended from "diagnostics," to include "prognostics" and more recently "theranostics." Theranostics describes a wide range of applications including the identification of a novel diagnostic marker that is used in order to identify patients for whom a newly developed drug will work.

\section{Cancer Biomarkers and Autoantibody Markers}

Cancer biomarkers have changed the way we detect and treat tumors. In recent years, the elucidation of several carcinogenic pathways, and a better understanding of tumor progression, has led to the identification of numerous tumor markers. The list of markers that have been identified is extensive and includes secreted proteins, transcription factors, and cell surface receptors. For example, $\alpha$-fetoprotein (AFP), a member of the albuminoid superfamily, is a cancer 
biomarker used for the monitoring of hepatoblastoma and hepatocellular carcinoma, as well as certain gastrointestinal cancers $[1,2]$. However, an increase of AFP is not always pathognomonic for liver cancer, as elevated AFP levels can be found in end-stage liver disease (cirrhosis) unrelated to tumor development [2, 3]. In fact, virtually all cancer biomarkers lack specificity to a particular tumor type and may be found in a variety of cancerous and noncancerous conditions. Hence, tumor markers alone are not diagnostic for cancer, and at present relatively few tumor markers are widely used by practicing physicians.

Autoantibodies targeting cellular constituents that act as autoantigens are useful serological markers for the diagnosis of autoimmune diseases and are also used in aiding cancer diagnosis [4-7]. Most of them are used for diagnostic purposes, and several of them show very good sensitivities and specificities for particular autoimmune diseases [4, 8-14]. Autoantibodies may also have prognostic significance, being able to identify individuals that will develop overt disease or patients at advanced stages of the disease and also those who will progress in a fast pace. The presence of autoantibodies may also identify patients who may have a better or poorer response to treatment and may also be used to monitor treatment response.

The great majority of tumor markers are not potent autoantibody markers and vice versa [15]. However, several studies have addressed the role of autoantibodies and, in particular, tumor-associated antigens (TAA), as targets of humoral and cellular immune responses [15-20]. Various autoantibody specificities have been described in patients with malignancy and some of those appear to be of diagnostic and prognostic significance, being able to allow early diagnosis or stratification of patients according to clinical phenotypes and disease outcome [18]. Investigations are being carried out for the delineation of autoantibody markers useful for the identification of individuals at risk for cancer [20]. Of particular interest are autoantibody markers of paraneoplastic neurological disorders characterised by highly specific autoantibodies directed against onconeuronal antigens [21-27]. Autoantibody panels and signature profiling with diagnostic or prognostic significance for various malignancies are under validation and may prove to be useful in the clinical setting [28]. The prevailing notion has been that antigens overexpressed in a state of a tumour act as cryptic antigens or neoantigens that are perceived as foreign from the immune system $[15,18]$. TAA are therefore capable of priming the immune system to recognize TAA and indeed tumor cells expressing them [15, 18]. On the other hand in conditions such as lymphomas, the degenerated B cells produce large amounts of autoantibodies that are insufficiently controlled by the peripheral regulatory machinery of the immune system.

The magnitude, duration, and efficacy of the TAA-specific CD4 and CD8 immune responses depend on several intrinsic factors [29]. An increasing number of studies are investigating ways to manipulate the efficacy of antigen-specific immune responses in a manner that can facilitate the eradication of antigen-expressing tissue-specific target cells [29].

The investigation of the fine specificity of the immune responses against specific TAA has led to the appreciation that some autoantibody specificities related to TAA may bear diagnostic and prognostic significance $[17,20]$. An increasing number of studies have obtained data to suggest that several TAA can be potential immunotherapeutic targets, in addition to aiding in the monitoring of disease progression and response to treatment [29-36]. There is no doubt that the study of humoral autoreactivity to TAA has helped investigators to estimate the sensitivity and specificity of individual anti-TAA antibodies. The role of TAA autoantibodies within the processes of carcinogenesis has been a topic of ongoing research. A systematic search of the literature published in 2009 has revealed more than 107 different TAA identified so far, most of which are of limited diagnostic relevance [37]. The majority of these TAA correspond to mutated or overexpressed antigens and were cytoplasmic proteins (42\%), while $26 \%$ corresponded to nuclear antigens and $21 \%$ to membranebound proteins [37]. Of interest, only $10 \%$ of the reported TAA corresponded to extracellular proteins such as secreted or extracellular matrix proteins [37].

In this review, we discuss the clinical significance of CUZD1, a novel biomarker with a dual role as a cancer and an autoantibody marker.

\section{CUZD1: Introduction to the Gene}

3.1. Terminology. CUZD1 stands for CUB and zona pellucidalike domains-containing protein 1 . The CUZD1 gene is also known as the uterine-ovarian-specific gene 44 (UO-44) and the estrogen-regulated gene 1 (ERG1).

3.2. Genomic Location. Human CUZD1 is mapped at chromosome 10q26.13 and contains nine exons. The loss of $10 \mathrm{q}$ is a frequent event in the development and progression of various malignant tumours including prostate adenocarcinoma, endometrial cancers, glioblastoma multiforme, and small cell lung cancer [38-42], suggesting the presence of several tumor suppressor genes in this chromosomal region, which are important for suppression of tumorigenesis and cancer progression.

The genomic structure of human CUZD1 is very similar to parts of the tumor suppressor DMBT1 (a gene deleted in malignant brain tumors) located at a locus exactly upstream of CUZD1 and in particular at 10q25.3-26.1 [43, 44]. The two proteins share a significant degree of homology (Figure 1).

3.3. Structure and Function. Human CUZD1 encodes for a 607 amino acids protein with a molecular weight of approximately $68 \mathrm{kDa}$. CUZD1 (as also the much larger DMBT1) contains two CUB domains and one ZP domain (Figure 2). The protein is highly conserved amongst species (Figure 3 ).

CUB (complement subcomponents $\mathrm{C} 1 \mathrm{r} / \mathrm{ClsC1s,} \mathrm{Uegf,}$ bone morphogenetic protein) domains [45] are structural motifs frequently present in various extracellular and plasma membrane-associated proteins, many of which are proteases $[46,47]$. Each consists of a catalytic domain and several CUB domains. CUB-containing proteins are multifunctional and play a role in embryogenenic signalling [48], complement activation [47, 49], inflammation and autoimmunity [50-52], 


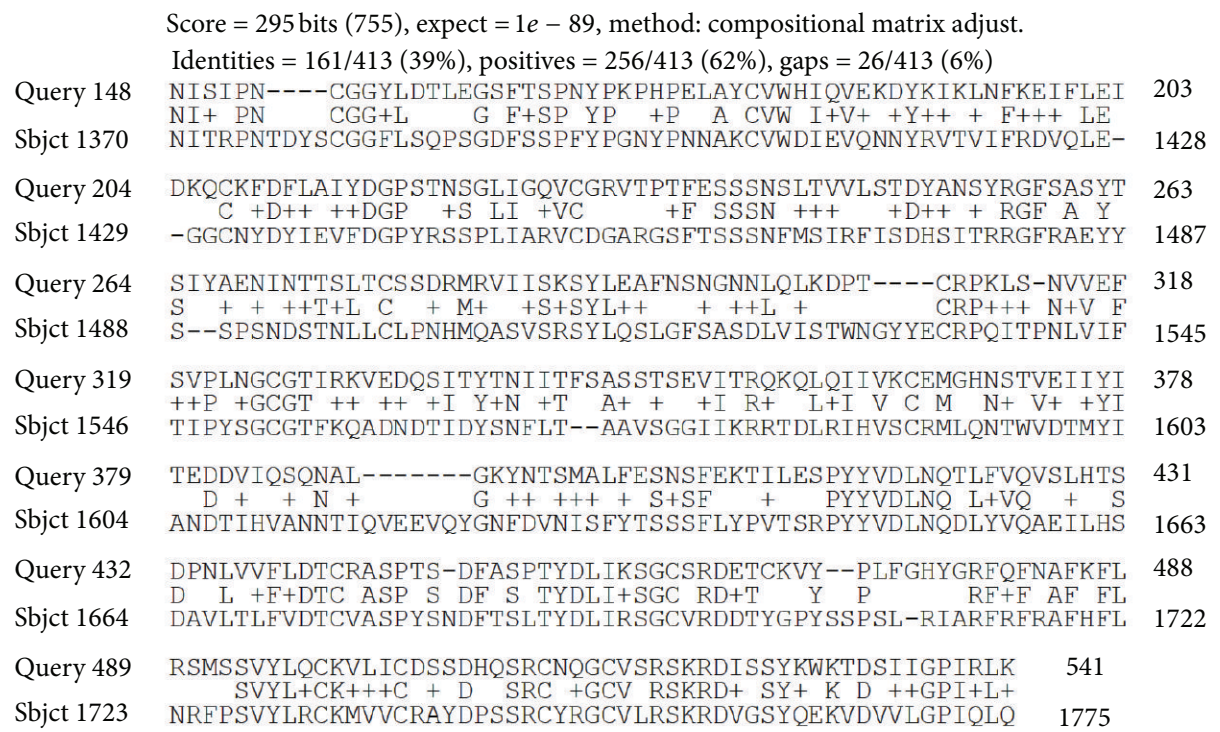

FIGURE 1: Amino acid comparison between human CUZD1 and DMBT1. There is a high degree of homology between the two proteins possibly reflecting the similarity between the CUB and ZP domains contained in both. The comparison has been performed using the BLASTp2 protein-protein programme. Top row: CUZD1; bottom row: DMTB1.

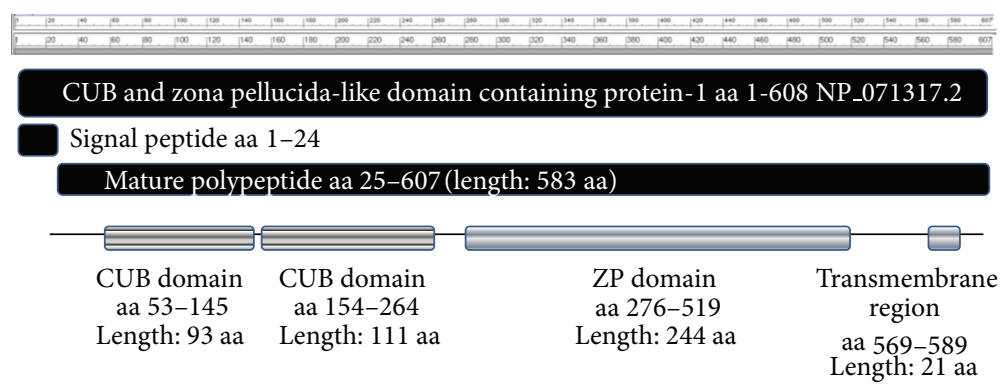

FIGURE 2: CUZD1 protein features including the CUB and ZP domain exact positioning within the protein.

cell adhesion and motility [53], cell migration and extracellular matrix degradation [54], cell signaling [53, 55, 56], axon guidance and neovascularization $[57,58]$, neurotransmission, and synaptic plasticity $[59,60]$. These proteins have also been involved in fertilization [61], thrombotic microangiopathy [55], cellular uptake and receptor-mediated endocytosis [62], and tumor suppression [51, 53, 63].

$\mathrm{ZP}$ glygoproteins (ZP1, ZP2, and ZP3) are responsible for sperm adhesion to the zona pellucida, an extracellular matrix surrounding oocytes [64]. ZP domains have been found in nonmammalian egg-coating multidomain transmembrane proteins such as tumor growth factor-beta receptor III $[64,65]$, major zymogen granule pancreatic glycoprotein GP2 [66-68], pancreatic ductal protein muclin, uromodulin (also known as Tamm-Horsfall protein) [69], inner ear protein $\beta$-tectorin, endoglin, no-mechanoreceptor potentialA (NompA), cuticlin-1, and intriguingly, in DMBT1 [70, 71].

\subsection{Tissue Expression of CUZD1}

3.4.1. CUZD1 in Uteri. In 1998, Kasik reported the isolation of a cDNA that was highly expressed in mouse uterus during late pregnancy [72]. At that time, he designated this cDNA as the UTCZP (uterine cub motif zona pellucida) motif [72]. That investigator found that the mRNA encoded by this gene is relatively abundant within the uterus. It first appears 6 days prior to birth and increases over subsequent days to reach maximal levels at 3 days prior to birth. mRNA expression levels then begin to suddenly decrease day by day at the last 3 days of pregnancy, and by the first day following birth it is practically undetectable. Expression of UTCZP mRNA is not found in nonpregnant uterus or in a variety of adult or fetal tissues including fetal and adult brain, thymus, spleen, pregnant maternal liver, fetal liver, adult liver, kidney, heart, and ovary.

In 1999, Chen et al. [73] reported a novel gene exhibiting $82 \%$ homology with mouse UTCZP. The expression of this gene induced in the uteri of ovariectomized or immature rats following administration of estrogen and was abolished upon anti-estrogen treatment. These authors termed this gene as estrogen-regulated gene 1 (ERG1; GenBank accession number: AF167170). They were able to show that estradiol treatment strongly induces ERG1 gene in rat uterus and oviduct and that its overexpression is restricted to surface 


\author{
CUZD1 Rat \\ CUZD1 Human \\ CUZD1 Chimpanzee \\ CUZD1 Monkey \\ CUZD1 Canine
}

CUZD1 Rat CUZD1 Human CUZD1 Chimpanzee CUZD1 Monkey CUZD1 Canine

CUZD1 Rat CUZD1 Human CUZD1 Chimpanzee CUZD1 Monkey CUZD1 Canine

CUZD1 Rat CUZD1 Human CUZD1 Chimpanzee CUZD1 Monkey CUZD1 Canine

CUZD1 Rat CUZD1 Human CUZD1 Chimpanzee CUZD1 Monkey CUZD1 Canine

CUZD1 Rat CUZD1 Human CUZD1 Chimpanzee CUZD1 Monkey CUZD1 Canine

CUZD1 Rat CUZD1 Human CUZD1 Chimpanzee CUZD1 Monkey CUZD1 Canine

CUZD1 Rat CUZD1 Human CUZD1 Chimpanzee CUZD1 Monkey CUZD1 Canine

CUZD1 Rat CUZD1 Human CUZD1 Chimpanzee CUZD1 Monkey CUZD1 Canine
MEVTGRLFIWAILAVSCRAQLNSTAAEGRPRCTASLGGANLGETHKALIL MELVRRLMPLTLLILSCLAELTMAEAEGNASCTVSLGGANMAETHKAMIL MELVRRLMPLTLLILSCLAELTMAEAEGNASCTVSLGGANMAETHKAMIL MELVKRLMPLILLILSCLAELTMAEAEGNSSCSVTLGGANMAETHKAMIL MEAVRRLVLLAFLMVPCLADLNLTKSEGKSSCQASLGGSSQSDAHRALIL

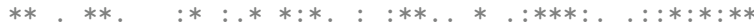

NLNADENCTWTIERPENRSIRIIFSHIQLDPDSRCENESIKVFDGRSTSG QLNPSENCTWTIERPENKSIRIIFSYVQLDPDGSCESENIKVFDGTSSNG QLNPSENCTWTIERPENKSIRIIFSYVQLDPDGSCESENIKVFDGTSSNG QLNPSENCTWTIERPENKSIRLIFSYIQLDPDGSCESENIKVFDGTSSNG NLHSNEDCTWTLQRPENKSIRIIFSYFQLDPDGPCESENIKVFDGNSTKA $: *: \ldots *: * * * *:: * * * *: * * *: * * *:, * * * * *, * *, *, * * * * * * * ;$.

PLLGEACSKNDFVPVFESSANSLTFQIVTDWTRVQRSVFIFYYFFSSGTT PLLGQVCSKNDYVPVFESSSSTLTFQIVTDSARIQRTVFVFYYFFSPNIS PLLGQVCSKNDYVPVFESSSSTLTFQIVTDSARIQRTVFVFYYFFSPNIS PLLGQVCSKNDYVPVFESSASTLTFQIVTDSARIQRTVFVFYYFFSPNTS SLLGKVCSKHDYIPVFESSSSTLTVQIVTDSVKTQRTVFIFYYFFSSGTS .***: .***:*::******: .:**.***** .: ***:**:******.. :

IPNCGGYLQTLEGSFSSPNYPRPHPELAYCVWHIQVEKGYKINLNFTELF IPNCGGYLDTLEGSFTSPNYPKPHPELAYCVWHIQVEKDYKIKLNFKEIF IPNCGGYLDTLEGSFTSPNYPKPHPELAYCVWHIQVEKDYKIKLNFKEIF IPNCGGYLDTLEGSFTSPNYPNPHPELAYCVWHIQVEKGYKIKLNFKEIF IPNCGGYLDSPKGSFTSPNYPNAHPALAYCVWHIRVEKGYKIKLNFRDIF **********: : : *** : ******.. *************:***,***:*** : : *

LEMDEYCRFDF IAVYDGPSTTSGLLKQVCGRGTPTFESSSDAMTVVLSTD LEIDKQCKFDFLAIYDGPSTNSGLIGQVCGRVTPTFESSSNSLTVVLSTD LEIDKQCKFDFLAIYDGPSTNSGLIGQVCGRVTPTFESSSNSLTVVLSTD LEIDKQCKFDFLAIYDGPSTNSGLIGQVCGRVTPTFESSSNSLTVVLSTD IEADEHCRFDF IAVYDGSSTTSGLMGQVCGYVKPTFESSSDSLTVVLSTD $: * *: *: * * *: *: * * *, * *, * * *: * * * *, * * * * * * *::: * * * * * * *$

YANSYRGFFASYASTYVQEVNTTSLSCASDKMRVIISKSYLQSLNYHESN YANSYRGFSASYTSIYAENINTTSLTCSSDRMRVIISKSYLEAFNSNGNN YANSYRGFSASYTSIYAENINTTSLTCSSDRMRVIISKSYLEAFNSNGNN YANSYRGFSASYTSIYAENINTTSLTCSSDRMRVIISKSYLEAFNSNGNT YANSYRGFSASYTSIYAENINTTSLTCSSDKMRIIINKSYLESFTYNENN $* * * * * * * * * * * * * * .::: * * * * *: *: * *: * *: * *, * * * *:::$ : $:$

LQLNDPTCRPSVSNVVEFSIPLHECGTIKKIEDHTISYTNIITFTQSPES LQLKDPTCRPKLSNVVEFSVPLNGCGTIRKVEDQSITYTNIITFSASSTS LQLKDPTCRPKLSNVVEFSIPLNGCGTIRKVEDQSITYTNIITFSASSTS LQLKDPTCRPKLSNVVEFSIPLNGCGTIRKVEDQSITYTNIITFSASSPS LQLNDPTCRPKISNVIDFSIPLDGCGTIKKVEDHSVTYTNIITLNPSPTS $* * *: * * * * * *,: * * *:: * *: * *, * * * *: *: * *:::: * * * * * *:, * * *$

AVITRKRHLQIVVTCEMEYNSTVEILYITEDDVIQNQSVLGKYNTSMALY EVITRQKQLQIIVKCEMGHNSTVEIIYITEDDVIQSQNALGKYNTSMALF EVITRQKQLQIILKCEMGHNSTVEIIYITEDDVIQSQNALGKYNTSMALF EVITRQKQLQIIVKCEMEHNSTVGLIYITEDDVIQNQNALGKYNTSMALF EVITRQKHLQIILKCEMEQNSTVEMMYITEDDIIQNESALGKYNTSMALF

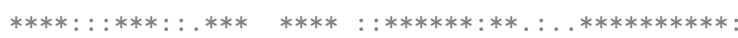

ESGSFENLIQESPYYVDLNQTLFVQATLHTSDPSLVVFLDTCRASPTSDF ESNSFEKTILESPYYVDLNQTLFVQVSLHTSDPNLVVFLDTCRASPTSDF ESNSFEKTILESPYYVDLNQTLFVQVSLHTSDPNLVVFLDTCRASPTSDF ESDSFEKTILESPYYVDLNQTLFVQVSLHTSDPNLVVFLDTCRASPTSDL ESSSFAKPILESPYYVDLNQTLFVQVSLYTSDPNLVVFLDTCRASPTPDF $* *, * *: * * * * * * * * * * * * * * * *,: *: * * * *, * * * * * * * * * * * * *, *:$

Figure 3: Continued. 


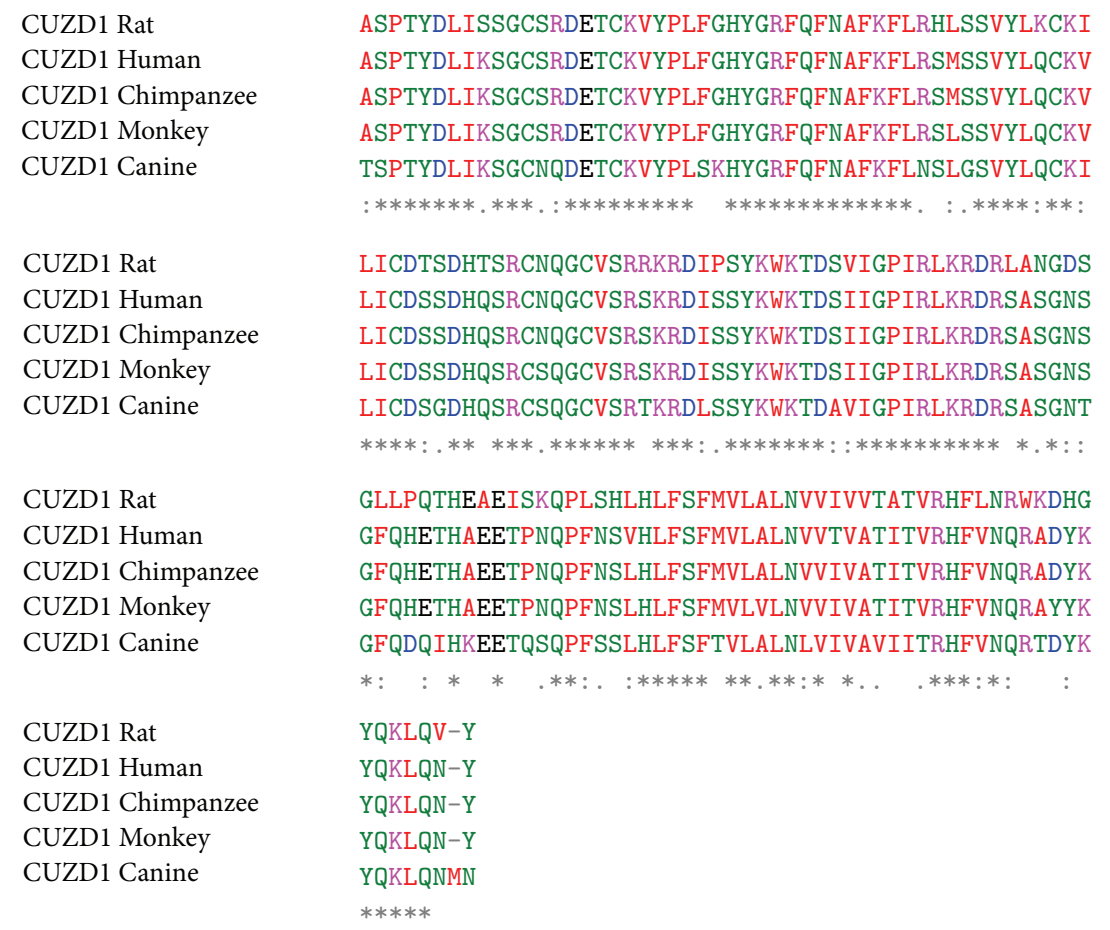

Figure 3: Multiple amino acid sequence alignment of CUZD1 of various species shows striking conservation. Sequences aligned include Rattus norvegicus (EDM 11686.1), Homo sapiens (NP_071317.2), Pan troglodytes (XP_001160209.2), Macaca fascicularis (EHH 65083.1), and Canis lupus familiaris (XP_544054.2). Asterisk indicates identities and semicolon indicates conserved or semiconserved substitutions. The alignment has been performed using the CLUSTAL W (1.83) multiple sequence alignment tool.

epithelium [73]. The expression level of ERG1 mRNA is high on day 1 of pregnancy, declined on day 2 , and was practically undetectable from days 3 to 6 of gestation. ERG1 expression was maximal at the proestrus stage of the ovarian cycle, coinciding with the estrogen-induced uterine cell proliferation, and clearly indicating that stage-specific manner of its expression during the ovarian cycle.

Two years later, Huynh et al. [74] reported the isolation of a uterine-ovarian-specific gene 44 (UO-44; GenBank accession number: AF022147) from a tamoxifen-induced rat uterine complementary DNA library in 2001. The UO-44 gene, currently known as CUZD1, was specifically expressed in the uterus and ovary of rats [74]. The rat UO-44 cDNA showed 99\% homology with the rat ERG1 cDNAs; thus the two genes were practically identical. In their study, Huynh et al. have shown that UO-44 mRNA was undetectable in uteri derived from OVX rats and was expressed after tamoxifen treatment [74]. In a set of in situ hybridization experiments on sections from uteri originated from control OVX and OVX-tamoxifen-treated rats, the same group of investigators have shown that while there was no UO-44 signal in uterus of OVX rats, high levels of UO-44 were detectable in the luminal epithelial cells and glandular population upon treatment with tamoxifen. Intriguingly, treatment with the pure antiestrogen ICI 182780 abrogated the effects of tamoxifen on the expression of UO-44, further suggesting that tamoxifen functions as an estrogen and induces UO-44 expression.
3.4.2. CUZD1 in Ovaries (and in Pancreas). Huynh et al. have obtained data clearly demonstrating that UO-44 mRNA is detectable in granulosa cells of ovaries [74]. They have also found varying amounts of UO-44 mRNA in granulosa cells of a mixed population of follicles. In particular, high levels of UO-44 expression were noted in the granulosa cells of medium-size follicles, while low-to-moderate UO44 expression was observed in granulosa cells of small and large follicles. Of interest, the UO-44 mRNA among granulosa cells within the same follicle was not uniform and greatly CUZD1 [75]. In a murine model of necrotizing pancreatitis, UTCZP- (CUZD1-) deficient mice developed more severe pancreatitis suggesting that CUZD1 may play an important role in trypsinogen activation and in the severity of pancreatitis [75].

\section{CUZD1: As a Cancer Biomarker}

One key experiment that suggested an important role for CUZD1 in carcinogenesis demonstrated that human UO44-specific antisera strikingly inhibit cell attachment and proliferation of NIH-OVCAR3 ovarian cancer cells [76]. This observation has led the authors to postulate that human UO44 may promote cell growth and facilitate invasion in ovarian cancer. The same group of investigators has shown that cisplatin treatment leads to the downregulation of human UO-44 expression and that silencing of human UO-44 based on sequence-specific siRNAs confers an enhanced sensitivity 
in cisplatin treatment of human ovarian cancer cells [77]. It also appears that UO-44 in ovarian cancer cells with overexpressed human UO-44 are also resistant to cisplatin [77]. In a recent study, Leung et al. [78] have measured CUZD1 levels in the serum of patients with various types of malignancies and healthy normal controls. Serum samples from patients with ovarian, breast, lung, colorectal, prostate, and testicular cancer were tested. Elevated levels of CUZD1 were found in patients with ovarian cancer, but also in breast and lung cancer [78]. However, this study was conducted in a very small number of sera and the diagnostic significance of CUZD1 as a cancer serum biomarker remains to be assessed in larger cohorts. Nevertheless, the latter study has shown that CUZD1 performed equally well as CA125 in two independent cohorts of samples consisting of healthy controls and ovarian cancer cases, and this may further suggest its potential role as a specific marker of ovarian cancer [78]. The authors reported that CUZD1 is a novel pancreatic cancer serum biomarker as well, but they did not present data to support this statement [78].

\section{Anti-CUZD1 Antibodies in Inflammatory Bowel Diseases}

Patients with Crohn's disease are characterised by the presence of organ-specific and nonorgan-specific autoantibodies [79-89]. Up to $30 \%$ of patients with Crohn's disease (CD) contain detectable pancreatic autoantibodies (PAB), giving either a droplet-like or a reticulogranular, cytoplasmic pattern $[52,84-87,90-93]$. The recognition of GP2 as the target of the droplet-like PABs in 2009 [94] has been followed by the recent identification of CUZD1 as the sole autoantigen of PABs giving the reticulogranular, cytoplasmic pattern by indirect immunofluorescence [91-93]. Several studies have attempted to delineate the diagnostic and clinical significance of anti-GP2 PAB [52, 90, 95-99], and the immunopathogenetic role of GP2 in CD has started to emerge [100-102]. However, the biological and clinical significance of humoral and cellular immune responses against CUZD1 remains poorly understood [91-93]. It has become apparent that the two autoreactivities rarely coexist and that the GP2 and CUZD1 are unlikely targets of cross-reactive autoantibodies.

The group of Winfried Stöcker has identified CUZD1 as the target of PABs giving the reticulogranular, cytoplasmic pattern (Figure 4) [103]. This group has also studied in some detail the diagnostic significance of anti-CUZD1 PABs in inflammatory bowel diseases [103].

5.1. CUZD1 as an Autoantigen. The first description of CUZD1 as autoantigenic target has been reported in the form of an abstract in 2008 and more recently as a full paper by Komorowski's group [103]. In a set of experiments, Komorowski et al. showed that the supernatant of homogenized human pancreas completely inhibits PAB reactivity, indicating that the $\mathrm{PAB}$ autoantigens are in the soluble fraction [103]. These investigators have tested the ability of various lectins to immobilize PAB-positive glycoproteins from cellfree human pancreas and used one of those, namely, UEAI (Ulex europaeus agglutinin I) to purify the glycoproteins using UEA-I affinity chromatography [103]. GP2 and CUZD1 were identified as the target autoantigens by mass spectrometry. Cloning and eukaryotic expression of CUZD1 has allowed the authors to assess the extent of anti-CUZD1 antibody reactivity using indirect immunofluorescence based on CUZD1 overexpressed human cell line HEK293 [103]. Absorption experiments showed that CUZD1 can completely abolish antibody reactivity of PABs giving the reticulogranular pattern, further indicating that CUZD1 is the only target of PABs giving this pattern [103].

5.2. Diagnostic Significance of Anti-CUZD1 Antibodies. Komorowski et al. reported anti-CUZD1 antibodies in $26 \%$ of patients with $\mathrm{CD}(19.8 \%$ alone and $6.2 \%$ with concomitant anti-GP2 antibodies) [103]. As expected, antiCUZD1 antibody reactivity was strongly correlated with the reticulogranular $\mathrm{PAB}$ pattern.

Kovacs et al. [104] used the same immunofluorescence approach to assess PAB reactivity against CUZD1 and GP2 in pediatric patients with $C D$. These authors did not provide data for individual reactivity to CUZD1 and GP2, but their overall data indicated the presence of PABs against the two antigens in $35.9 \%$ of patients with CD, $24.5 \%$ of patients with ulcerative colitis, and in none of the pediatric controls. The unexpectedly high prevalence of anti-CUZD1 and anti-GP2 antibodies in patients with ulcerative colitis warrants further investigations, as PABs are only found in less than $8 \%$ of patients with this disease.

More recently, Roggenbuck et al. have also assessed the prevalence of anti-CUZD1 antibodies in their cohort and found that these autoantibodies are present in $29.2 \%$ of patients with IBD and, in particular, in $22.6 \%$ and $14.9 \%$ of patients with CD and UC, respectively [105].

Our own preliminary data in a large cohort of patients with IBD tested by the same technique indicates the presence of IgA or IgG anti-CUZD1 in $21.7 \%$ CD compared to $10.8 \%$ UC patients (Bogdanos et al., unpublished data).

5.3. Clinical Significance of Anti-CUZD1 Antibodies. Currently, there are no data evaluating the clinical relevance of anti-CUZD1 antibodies in patients with inflammatory bowel diseases.

5.4. The Role of CUZD1 in the Immunopathogenesis of Crohn's Disease. The exact role of CUZD1 in the processes that take place prior to the induction of immune-mediated intestinal destruction remains elusive. Komorowski et al. [103] speculated that CUZD1 is released from the pancreas and participates in the innate immune responses that affect the intestinal lumen. The involvement of the $\mathrm{ZP}$ domain of CUZD1 may be crucial for the development of intestinal damage, as ZP domains polymerize under specific environmental conditions leading to the aggregation of bacteria preventing their adhesion to mucosal cells and CUZD1 autoantibodies may interfere with such a process [103]. The de novo induction of anti-CUZD1 antibodies can be the end result of microbialinduced autoimmunity, similar to that possibly involved in the induction of GP2 autoantibodies $[100,101]$. As anti-GP2 


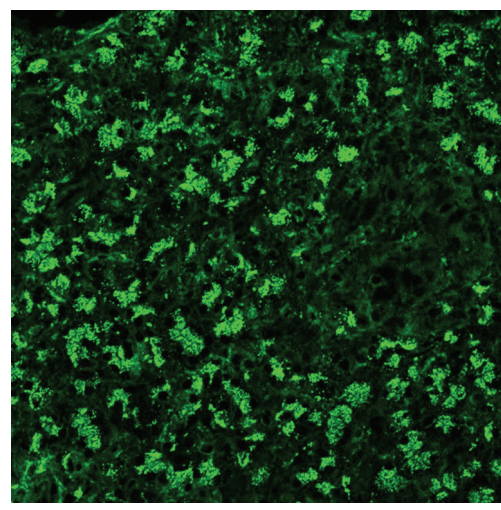

(a)

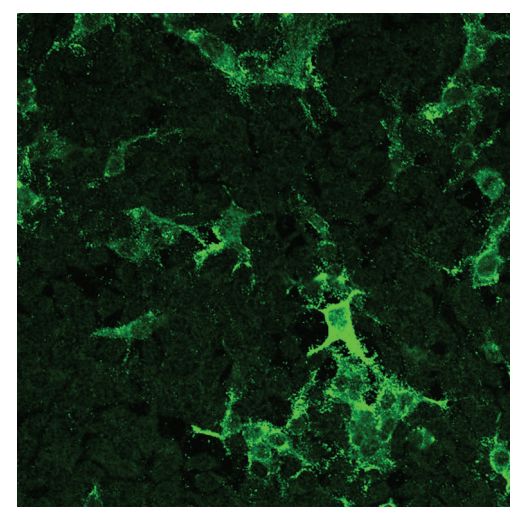

(b)

FIGURE 4: Immunofluorescence staining patterns of CUZD1-specific pancreatic autoantibodies giving a reticulogranular pattern using pancreatic tissue (a) or CUZD1-overexressed HEK3 cells (b).

and anti-CUZD1 antibodies infrequently cooccur, it may be argued that the mechanisms that lead to the induction of these autoantibodies differ [52].

\section{Conclusion}

CUZD1 is a pancreatic antigen with a dual role, as a cancer biomarker and an autoantibody target. This antigen is one of the very few that may play a role in distinct pathological entities, such as ovarian cancer and Crohn's disease, for reasons poorly understood. The involvement of CUZD1 in carcinogenesis warrants further investigation. We also have to understand the role of CUZD1 in the innate and adaptive immune responses characteristic of CD [52]. We anticipate that the pathogenic role of this antigen will be delineated in the years to come.

\section{Abbreviations}

AFP: $\quad \alpha$-fetoprotein

CUB: Complement subcomponents $\mathrm{Clr} / \mathrm{ClsC}$ s, uegf, bone morphogenetic protein

CUZD1: CUB and zona pellucida-like domains-containing protein 1

DMBT1: Deleted in malignant brain tumors

ERG1: Estrogen regulated gene 1

IBD: Inflammatory bowel diseases

PAB: Pancreatic autoantibody

TAA: Tumor associated antigens

UO-44: Uterine-ovarian-specific gene 44

UTCZP: UTerine Cub motif Zona Pellucida

ZP: $\quad$ Zona pellucida.

\section{Acknowledgments}

The authors thank Dr Lars Komorowski (EUROIMMUN) for providing the slides overexpressing CUZD1 and for critical reading of the paper.

\section{References}

[1] D. Y. Kim, Y. H. Paik, S. H. Ahn et al., "PIVKA-II is a useful tumor marker for recurrent hepatocellular carcinoma after surgical resection," Oncology, vol. 72, no. 1, pp. 52-57, 2007.

[2] S. B. Paul, M. S. Gulati, V. Sreenivas et al., "Evaluating patients with cirrhosis for hepatocellular carcinoma: value of clinical symptomatology, imaging and alpha-fetoprotein," Oncology, vol. 72, no. 1, pp. 117-123, 2007.

[3] S. Gupta, S. Bent, and J. Kohlwes, “Test characteristics of alphafetoprotein for detecting hepatocellular carcinoma in patients with hepatitis C. A systematic review and critical analysis," Annals of Internal Medicine, vol. 139, no. 1, pp. 46-50, 2003.

[4] E. M. Tan, "Autoantibodies, autoimmune disease, and the birth of immune diagnostics," Journal of Clinical Investigation, vol. 122, no. 11, pp. 3835-3836, 2012.

[5] Y. Yao, Y. Fan, J. Wu et al., "Potential application of non-small cell lung cancer-associated autoantibodies to early cancer diagnosis," Biochemical and Biophysical Research Communications, vol. 423, no. 3, pp. 613-619, 2012.

[6] Q. Shao, P. Ren, Y. Li et al., "Autoantibodies against glucoseregulated protein 78 as serological diagnostic biomarkers in hepatocellular carcinoma," International Journal of Oncology, vol. 41, no. 3, pp. 1061-1067, 2012.

[7] R. Kiyamova, O. Garifulin, V. Gryshkova et al., "Preliminary study of thyroid and colon cancers-associated antigens and their cognate autoantibodies as potential cancer biomarkers," Biomarkers, vol. 17, no. 4, pp. 362-371, 2012.

[8] F. J. Quintana, A. Yeste, H. L. Weiner, and R. Covacu, "Lipids and lipid-reactive antibodies as biomarkers for multiple sclerosis," Journal of Neuroimmunology, vol. 248, no. 1-2, pp. 53-57, 2012.

[9] M. Gold, R. Pul, J. P. Bach, M. Stangel, and R. Dodel, "Pathogenic and physiological autoantibodies in the central nervous system," Immunological Reviews, vol. 248, no. 1, pp. 68-86, 2012.

[10] D. P. Bogdanos, P. Invernizzi, I. R. Mackay, and D. Vergani, "Autoimmune liver serology: current diagnostic and clinical challenges," World Journal of Gastroenterology, vol. 14, no. 21, pp. 3374-3387, 2008.

[11] D. P. Bogdanos, G. Mieli-Vergani, and D. Vergani, "Autoantibodies and their antigens in autoimmune hepatitis," Seminars in Liver Disease, vol. 29, no. 3, pp. 241-253, 2009. 
[12] D. S. Smyk, E. I. Rigopoulou, A. L. Koutsoumpas, S. Kriese, A. K. Burroughs, and D. P. Bogdanos, "Autoantibodies in autoimmune pancreatitis," International Journal of Rheumatology, vol. 2012, Article ID 940831, 8 pages, 2012.

[13] Y. Hamaguchi, "Autoantibody profiles in systemic sclerosis: predictive value for clinical evaluation and prognosis," Journal of Dermatology, vol. 37, no. 1, pp. 42-53, 2010.

[14] N. J. Olsen, Q. Z. Li, J. Quan, L. Wang, A. Mutwally, and D. R. Karp, "Autoantibody profiling to follow evolution of lupus syndromes," Arthritis Research \& Therapy, vol. 14, no. 4, Article ID R174, 2012.

[15] E. M. Tan, "Autoantibodies as reporters identifying aberrant cellular mechanisms in tumorigenesis," Journal of Clinical Investigation, vol. 108, no. 10, pp. 1411-1415, 2001.

[16] H. Lu, V. Goodell, and M. L. Disis, "Humoral immunity directed against tumor-associated antigens as potential biomarkers for the early diagnosis of cancer," Journal of Proteome Research, vol. 7, no. 4, pp. 1388-1394, 2008.

[17] W. Liu, B. Peng, Y. Lu, W. Xu, W. Qian, and J. Y. Zhang, "Autoantibodies to tumor-associated antigens as biomarkers in cancer immunodiagnosis," Autoimmunity Reviews, vol. 10, no. 6, pp. 331-335, 2011.

[18] E. M. Tan and J. Zhang, "Autoantibodies to tumor-associated antigens: reporters from the immune system," Immunological Reviews, vol. 222, no. 1, pp. 328-340, 2008.

[19] H. T. Tan, J. Low, S. G. Lim, and M. C. M. Chung, "Serum autoantibodies as biomarkers for early cancer detection," FEBS Journal, vol. 276, no. 23, pp. 6880-6904, 2009.

[20] F. F. Madrid and M. C. Maroun, "Serologic laboratory findings in malignancy," Rheumatic Disease Clinics of North America, vol. 37, no. 4, pp. 507-525, 2011.

[21] F. Blaes and M. Tschernatsch, "Paraneoplastic neurological disorders," Expert Review of Neurotherapeutics, vol. 10, no. 10, pp. 1559-1568, 2010.

[22] A. Saiz, J. Dalmau, M. H. Butler et al., "Anti-amphiphysin I antibodies in patients with paraneoplastic neurological disorders associated with small cell lung carcinoma," Journal of Neurology Neurosurgery and Psychiatry, vol. 66, no. 2, pp. 214-217, 1999.

[23] J. Dalmau, S. H. Gultekin, R. Voltz et al., "Mal, a novel neuron- and testis-specific protein, is recognized by the serum of patients with paraneoplastic neurological disorders," Brain, vol. 122, no. 1, pp. 27-39, 1999.

[24] A. Vincent, J. Honnorat, J. C. Antoine, B. Giometto, J. Dalmau, and B. Lang, "Autoimmunity in paraneoplastic neurological disorders," Journal of Neuroimmunology, vol. 84, no. 1, pp. 105109, 1998.

[25] J. W. B. Moll, S. C. Henzen-Logmans, and C. J. Vecht, "Antineuronal antibodies in paraneoplastic neurological disorders with small-cell lung carcinoma," Clinical Neurology and Neurosurgery, vol. 92, no. 3, pp. 223-228, 1990.

[26] V. A. Lennon, "Anti-Purkinje cell cytoplasmic and neuronal nuclear antibodies aid diagnosis of paraneoplastic autoimmune neurological disorders," Journal of Neurology Neurosurgery and Psychiatry, vol. 52, no. 12, pp. 1438-1439, 1989.

[27] W. Grisold, M. Drlicek, U. Liszka, and W. Popp, "Anti-purkinje cell antibodies are specific for small-cell lung cancer but not for paraneoplastic neurological disorders," Journal of Neurology, vol. 236, no. 1, p. 64, 1989.

[28] W. Chang, L. Wu, F. Cao et al., "Development of autoantibody signatures as biomarkers for early detection of colorectal carcinoma," Clinical Cancer Research, vol. 17, no. 17, pp. 5715-5724, 2011.
[29] N. Schmidt, C. Neumann-Haefelin, and R. Thimme, "Cellular immune responses to hepatocellular carcinoma: lessons for immunotherapy," Digestive Diseases, vol. 30, no. 5, pp. 483-491, 2012.

[30] J. Arnason and D. Avigan, "Evolution of cellular immunotherapy: from allogeneic transplant to dendritic cell vaccination as treatment for multiple myeloma," Immunotherapy, vol. 4, no. 10, pp. 1043-1051, 2012.

[31] D. Nobuoka, T. Yoshikawa, M. Takahashi et al., "Intratumoral peptide injection enhances tumor cell antigenicity recognized by cytotoxic T lymphocytes: a potential option for improvement in antigen-specific cancer immunotherapy," Cancer Immunology, Immunotherapy, 2012.

[32] S. Hong, H. Li, J. Qian, J. Yang, Y. Lu, and Q. Yi, “Optimizing dendritic cell vaccine for immunotherapy in multiple myeloma: tumour lysates are more potent tumour antigens than idiotype protein to promote anti-tumour immunity," Clinical \& Experimental Immunology, vol. 170, no. 2, pp. 167-177, 2012.

[33] S. Anguille, V. F. Van Tendeloo, and Z. N. Berneman, "Leukemia-associated antigens and their relevance to the immunotherapy of acute myeloid leukemia," Leukemia, vol. 26, no. 10, pp. 2186-2196, 2012.

[34] E. L. J. M. Smits, Z. N. Berneman, and V. F. I. Van Tendeloo, "Immunotherapy of acute myeloid leukemia: current approaches," Oncologist, vol. 14, no. 3, pp. 240-252, 2009.

[35] D. H. Palmer, R. S. Midgley, N. Mirza et al., "A phase II study of adoptive immunotherapy using dendritic cells pulsed with tumor lysate in patients with hepatocellular carcinoma," Нераtology, vol. 49, no. 1, pp. 124-132, 2009.

[36] M. A. Neller, J. A. López, and C. W. Schmidt, "Antigens for cancer immunotherapy," Seminars in Immunology, vol. 20, no. 5, pp. 286-295, 2008.

[37] M. Reuschenbach, M. von Knebel Doeberitz, and N. Wentzensen, "A systematic review of humoral immune responses against tumor antigens," Cancer Immunology, Immunotherapy, vol. 58, no. 10, pp. 1535-1544, 2009.

[38] S. L. Peiffer, T. J. Herzog, D. J. Tribune, D. G. Mutch, D. J. Gersell, and P. J. Goodfellow, "Allelic loss of sequences from the long arm of chromosome 10 and replication errors in endometrial cancers," Cancer Research, vol. 55, no. 9, pp. 1922-1926, 1995.

[39] B. K. A. Rasheed, R. E. McLendon, H. S. Friedman et al., "Chromosome 10 deletion mapping in human gliomas: a common deletion region in 10q25," Oncogene, vol. 10, no. 11, pp. 22432246, 1995.

[40] I. C. Gray, S. M. A. Phillips, S. J. Lee, J. P. Neoptolemos, J. Weissenbach, and N. K. Spurr, "Loss of the chromosomal region 10q23-25 in prostate cancer," Cancer Research, vol. 55, no. 21, pp. 4800-4803, 1995.

[41] S. K. Kim, J. Y. Ro, B. L. Kemp et al., "Identification of two distinct tumor-suppressor loci on the long arm of chromosome 10 in small cell lung cancer," Oncogene, vol. 17, no. 13, pp. 1749-1753, 1998.

[42] R. P. T. Somerville, Y. Shoshan, C. Eng, G. Barnett, D. Miller, and J. K. Cowell, "Molecular analysis of two putative tumour suppressor genes, PTEN and DMBT, which have been implicated in glioblastoma multiforme disease progression," Oncogene, vol. 17, no. 13, pp. 1755-1757, 1998.

[43] J. Mollenhauer, S. Wiemann, W. Scheurlen et al., "DMBT1, a new member of the SRCR superfamily, on chromosome 10q25.3-26.1 is deleted in malignant brain tumours," Nature Genetics, vol. 17, no. 1, pp. 32-39, 1997. 
[44] E. Lualdi, E. Chiariello, and G. Finocchiaro, "Identification of regulatory regions of the putative tumor suppressor gene DMBT1," Biochemical and Biophysical Research Communications, vol. 270, no. 2, pp. 673-675, 2000.

[45] P. Bork and G. Beckmann, "The CUB domain. A widespread module in developmentally regulated proteins," Journal of Molecular Biology, vol. 231, no. 2, pp. 539-545, 1993.

[46] I. Bally, V. Rossi, T. Lunardi, N. M. Thielens, C. Gaboriaud, and G. J. Arlaud, "Identification of the Clq-binding sites of human $\mathrm{Clr}$ and C1s. A refined three-dimensional model of the C1 complex of complement," Journal of Biological Chemistry, vol. 284, no. 29, pp. 19340-19348, 2009.

[47] C. Gaboriaud, N. M. Thielens, L. A. Gregory, V. Rossi, J. C. Fontecilla-Camps, and G. J. Arlaud, "Structure and activation of the C1 complex of complement: unraveling the puzzle," Trends in Immunology, vol. 25, no. 7, pp. 368-373, 2004.

[48] H. X. Lee, A. L. Ambrosio, B. Reversade, and E. M. De Robertis, "Embryonic dorsal-ventral signaling: secreted Frizzled-related proteins as inhibitors of tolloid proteinases," Cell, vol. 124, no. 1, pp. 147-159, 2006.

[49] R. Sørensen, S. Thiel, and J. C. Jensenius, "Mannan-bindinglectin-associated serine proteases, characteristics and disease associations," Springer Seminars in Immunopathology, vol. 27, no. 3, pp. 299-319, 2005.

[50] C. M. Milner and A. J. Day, "TSG-6: a multifunctional protein associated with inflammation," Journal of Cell Science, vol. 116, no. 10, pp. 1863-1873, 2003.

[51] W. Kang and K. B. M. Reid, "DMBT1, a regulator of mucosal homeostasis through the linking of mucosal defense and regeneration?" FEBS Letters, vol. 540, no. 1-3, pp. 21-25, 2003.

[52] D. P. Bogdanos, E. I. Rigopoulou, D. S. Smyk et al., "Diagnostic value, clinical utility and pathogenic significance of reactivity to the molecular targets of Crohn's disease specific-pancreatic autoantibodies," Autoimmunity Reviews, vol. 11, no. 2, pp. 143$148,2011$.

[53] C. H. Benes, G. Poulogiannis, L. C. Cantley, and S. P. Soltoff, "The SRC-associated protein CUB Domain-Containing Protein-1 regulates adhesion and motility," Oncogene, vol. 31, no. 5, pp. 653-663, 2012.

[54] Y. Miyazawa, T. Uekita, N. Hiraoka et al., "CUB domaincontaining protein 1 , a prognostic factor for human pancreatic cancers, promotes cell migration and extracellular matrix degradation," Cancer Research, vol. 70, no. 12, pp. 5136-5146, 2010.

[55] Z. Tao, Y. Peng, L. Nolasco et al., "Recombinant CUB-1 domain polypeptide inhibits the cleavage of ULVWF strings by ADAMTS13 under flow conditions," Blood, vol. 106, no. 13, pp. 4139-4145, 2005.

[56] G. Davidson, B. Mao, I. del Barco Barrantes, and C. Niehrs, "Kremen proteins interact with Dickkopf1 to regulate anteroposterior CNS patterning," Development, vol. 129, no. 24, pp. 5587-5596, 2002.

[57] G. Neufeld, T. Cohen, N. Shraga, T. Lange, O. Kessler, and Y. Herzog, "The neuropilins: multifunctional semaphorin and VEGF receptors that modulate axon guidance and angiogenesis," Trends in Cardiovascular Medicine, vol. 12, no. 1, pp. 13-19, 2002.

[58] C. Gu, B. J. Limberg, G. Brian Whitaker et al., "Characterization of neuropilin-1 structural features that confer binding to semaphorin $3 \mathrm{~A}$ and vascular endothelial growth factor 165 ,"
Journal of Biological Chemistry, vol. 277, no. 20, pp. 18069-18076, 2002.

[59] D. Ng, G. M. Pitcher, R. K. Szilard et al., "Netol is a novel CUB-domain NMDA receptor-interacting protein required for synaptic plasticity and learning," PLoS Biology, vol. 7, no. 2, article e41, 2009.

[60] Y. Zheng, J. E. Mellem, P. J. Brockie, D. M. Madsen, and A. V. Maricq, "SOL-1 is a CUB-domain protein required for GLR-1 glutamate receptor function in C. elegans," Nature, vol. 427, no. 6973, pp. 451-457, 2004.

[61] E. Töpfer-Petersen, A. Romcro, P. F. Varcla et al., "Spermadhesins: a new protein family. Facts, hypotheses and perspectives," Andrologia, vol. 30, no. 4-5, pp. 217-224, 1998.

[62] T. Sugiyama, H. Kumagai, Y. Morikawa et al., "A novel lowdensity lipoprotein receptor-related protein mediating cellular uptake of apolipoprotein E-enriched $\beta$-VLDL in vitro," Biochemistry, vol. 39, no. 51, pp. 15817-15825, 2000.

[63] C. F. Hooi, C. Blancher, W. Qiu et al., "ST7-mediated suppression of tumorigenicity of prostate cancer cells is characterized by remodeling of the extracellular matrix," Oncogene, vol. 25, no. 28, pp. 3924-3933, 2006.

[64] P. Bork and C. Sander, "A large domain common to sperm receptors (Zp2 and $\mathrm{Zp} 3$ ) and TGF- $\beta$ type III receptor," FEBS Letters, vol. 300, no. 3, pp. 237-240, 1992.

[65] F. López-Casillas, S. Cheifetz, J. Doody, J. L. Andres, W. S. Lane, and J. Massagué, "Structure and expression of the membrane proteoglycan betaglycan, a component of the TGF- $\beta$ receptor system," Cell, vol. 67, no. 4, pp. 785-795, 1991.

[66] T. C. Hoops and M. J. Rindler, "Isolation of the cDNA encoding glycoprotein-2 (GP-2), the major zymogen granule membrane protein: homology to uromodulin/Tamm-Horsfall protein," Journal of Biological Chemistry, vol. 266, no. 7, pp. 4257-4263, 1991.

[67] M. J. Rindler and T. C. Hoops, "The pancreatic membrane protein GP-2 localizes specifically to secretory granules and is shed into the pancreatic juice as a protein aggregate," European Journal of Cell Biology, vol. 53, no. 1, pp. 154-163, 1990.

[68] S. I. Fukuoka, S. D. Freedman, H. Yu, V. P. Sukhatme, and G. A. Scheele, "GP-2/THP gene family encodes self-binding glycosylphosphatidylinositol-anchored proteins in apical secretory compartments of pancreas and kidney," Proceedings of the National Academy of Sciences of the United States of America, vol. 89, no. 4, pp. 1189-1193, 1992.

[69] D. Pennica, W. J. Kohr, and W. J. Kuang, "Identification of human uromodulin as the Tamm-Horsfall urinary glycoprotein," Science, vol. 236, no. 4797, pp. 83-88, 1987.

[70] L. Jovine, C. C. Darie, E. S. Litscher, and P. M. Wassarman, "Zona pellucida domain proteins," Annual Review of Biochemistry, vol. 74, pp. 83-114, 2005.

[71] L. Jovine, H. Qi, Z. Williams, E. S. Litscher, and P. M. Wassarman, "A duplicated motif controls assembly of zona pellucida domain proteins," Proceedings of the National Academy of Sciences of the United States of America, vol. 101, no. 16, pp. 59225927, 2004.

[72] J. W. Kasik, "A cDNA cloned from pregnant mouse uterus exhibits temporo-spatial expression and predicts a novel protein," Biochemical Journal, vol. 330, no. 2, pp. 947-950, 1998.

[73] D. Chen, X. Xu, Z. Li-Ji et al., "Cloning and uterus/oviductspecific expression of a novel estrogen- regulated gene (ERG1)," Journal of Biological Chemistry, vol. 274, no. 45, pp. 3221532224, 1999. 
[74] H. Huynh, C. Y. Ng, K. B. Lim et al., "Induction of UO-44 gene expression by tamoxifen in the rat uterus and ovary," Endocrinology, vol. 142, no. 7, pp. 2985-2995, 2001.

[75] T. Imamura, M. Asada, S. K. Vogt, D. A. Rudnick, M. E. Lowe, and L. J. Muglia, "Protection from pancreatitis by the zymogen granule membrane protein integral membrane-associated protein-1," Journal of Biological Chemistry, vol. 277, no. 52, pp. 50725-50733, 2002.

[76] C. T. C. Leong, Y. N. Chuan, P. N. Chee et al., "Molecular cloning, characterization and isolation of novel spliced variants of the human ortholog of a rat estrogen-regulated membraneassociated protein, UO-44," Oncogene, vol. 23, no. 33, pp. 57075718, 2004.

[77] C. T. C. Leong, C. K. Ong, S. K. Tay, and H. Huynh, "Silencing expression of UO-44 (CUZD1) using small interfering RNA sensitizes human ovarian cancer cells to cisplatin in vitro," Oncogene, vol. 26, no. 6, pp. 870-880, 2007.

[78] F. Leung, A. Soosaipillai, V. Kulasingam, and E. P. Diamandis, "CUB and zona pellucida-like domain-containing protein 1 (CUZD1): a novel serological biomarker for ovarian cancer," Clinical Biochemistry, vol. 45, no. 18, pp. 1543-1546, 2012.

[79] X. Bossuyt, "Serologic markers in inflammatory bowel disease," Clinical Chemistry, vol. 52, no. 2, pp. 171-181, 2006.

[80] K. Conrad, H. Schmechta, A. Klafki et al., "Serological differentiation of inflammatory bowel diseases," European Journal of Gastroenterology and Hepatology, vol. 14, no. 2, pp. 129-135, 2002.

[81] B. Desir, D. K. Amre, S. E. Lu et al., "Utility of serum antibodies in determining clinical course in pediatric Crohn's disease," Clinical Gastroenterology and Hepatology, vol. 2, no. 2, pp. 139146, 2004.

[82] H. Fricke, A. Birkhofer, C. Folwaczny, W. Meister, and P. C. Scriba, "Characterization of antigens from the human exocrine pancreatic tissue (Pag) relevant as target antigens for autoantibodies in Crohn's disease," European Journal of Clinical Investigation, vol. 29, no. 1, pp. 41-45, 1999.

[83] S. Joossens, W. Reinisch, S. Vermeire et al., "The value of serologic markers in indeterminate colitis: a prospective follow-up study," Gastroenterology, vol. 122, no. 5, pp. 1242-1247, 2002.

[84] S. Joossens, S. Vermeire, K. Van Steen et al., "Pancreatic autoantibodies in inflammatory bowel disease," Inflammatory Bowel Diseases, vol. 10, no. 6, pp. 771-777, 2004.

[85] F. H. Klebl, F. Bataille, C. Huy, F. Hofstädter, J. Schölmerich, and G. Rogler, "Association of antibodies to exocrine pancreas with subtypes of Crohn's disease," European Journal of Gastroenterology and Hepatology, vol. 17, no. 1, pp. 73-77, 2005.

[86] P. L. Lakatos, I. Aitorjay, T. Szamosi et al., "Pancreatic autoantibodies are associated with reactivity to microbial antibodies, penetrating disease behavior, perianal disease, and extraintestinal manifestations, but not with NOD2/CARD15 or TLR4 genotype in a Hungarian IBD cohort," Inflammatory Bowel Diseases, vol. 15, no. 3, pp. 365-374, 2009.

[87] S. Desplat-Jégo, C. Johanet, A. Escande et al., "Update in AntiSaccharomyces cerevisiae antibodies, anti-nuclear associated anti-neutrophil antibodies and antibodies to exocrine pancreas detected by indirect immunofluorescence as biomarkers in chronic inflammatory bowel diseases: results of a multicenter study," World Journal of Gastroenterology, vol. 13, no. 16, pp. 2312-2318, 2007.

[88] D. Roggenbuck, D. Reinhold, T. Wex et al., "Letter: the authors' reply," Gut, vol. 61, no. 1, pp. 164-165, 2012.
[89] N. Vermeulen, K. O. De Béeck, S. Vermeire et al., "Identification of a novel autoantigen in inflammatory bowel disease by protein microarray," Inflammatory Bowel Diseases, vol. 17, no. 6, pp. 1291-1300, 2011.

[90] K. Op De Beeck, S. Vermeire, P. Rutgeerts, and X. Bossuyt, "Antibodies to GP2, the major zymogen granule membrane glycoprotein, in inflammatory bowel diseases," Gut, vol. 61, pp. 162-164, 2012.

[91] W. Stöcker, M. Otte, S. Ulrich et al., "Autoimmunity to pancreatic juice in Crohn's disease. Results of an autoantibody screening in patients with chronic inflammatory bowel disease," Scandinavian Journal of Gastroenterology, vol. 139, pp. 41-52, 1987.

[92] W. Stöcker, M. Otte, S. Ulrich, D. Normann, K. Stöcker, and G. Jantschek, "Autoantibodies against the exocrine pancreas and against intestinal goblet cells in the diagnosis of Crohn's disease and ulcerative colitis," Deutsche Medizinische Wochenschrift, vol. 109, pp. 1963-1969, 1984.

[93] W. Stöcker, B. Teegen, C. Probst et al., "CUZD1 and GP2 are the exocrine pancreas autoantigens in Crohn's disease," in From Pathogenesis to Therapy of Autoimmune Diseases, Pabst Science Publishers, Lengerich, Germany, 2009.

[94] D. Roggenbuck, G. Hausdorf, L. Martinez-Gamboa et al., "Identification of GP2, the major zymogen granule membrane glycoprotein, as the autoantigen of pancreatic antibodies in Crohn's disease," Gut, vol. 58, no. 12, pp. 1620-1628, 2009.

[95] P. Pavlidis, O. Romanidou, D. Roggenbuck et al., "Ileal inflammation may trigger the development of GP2-specific pancreatic autoantibodies in patients with Crohn's disease," Clinical and Developmental Immunology, vol. 2012, Article ID 640835, 8 pages, 2012.

[96] P. Pavlidis, A. Forbes, and D. P. Bogdanos, "Antibodies to glycoprotein 2 (GP2) in patients with inflammatory bowel diseases from UK," Clinica Chimica Acta, vol. 412, no. 11-12, pp. 11631164, 2011.

[97] D. Roggenbuck, D. Reinhold, T. Wex et al., "Autoantibodies to GP2, the major zymogen granule membrane glycoprotein, are new markers in Crohn's disease," Clinica Chimica Acta, vol. 412, no. 9-10, pp. 718-724, 2011.

[98] D. Roggenbuck, R. L. Humbel, D. Reinhold, D. P. Bogdanos, K. Conrad, and M. W. Laass, "Glycoprotein 2 antibodies in inflammatory bowel disease-no association with disease phenotype?" Journal of Pediatric Gastroenterology and Nutrition, vol. 56, no. 1, article e5, 2013.

[99] D. P. Bogdanos, D. Roggenbuck, D. Reinhold et al., "Pancreaticspecific autoantibodies to glycoprotein 2 mirror disease location and behaviour in younger patients with Crohn's disease," BMC Gastroenterology, vol. 12, article 102, 2012.

[100] K. Hase, K. Kawano, T. Nochi et al., "Uptake through glycoprotein 2 of FimH + bacteria by M cells initiates mucosal immune response," Nature, vol. 462, no. 7270, pp. 226-230, 2009.

[101] H. Ohno and K. Hase, "Glycoprotein 2 (GP2) grabbing the fimH+ bacteria into $\mathrm{m}$ cells for mucosal immunity," Gut Microbes, vol. 1, no. 6, pp. 407-410, 2010.

[102] M. A. Hölzl, J. Hofer, J. J. Kovarik et al., “The zymogen granule protein 2 (GP2) binds to scavenger receptor expressed on endothelial cells I (SREC-I)," Cellular Immunology, vol. 267, no. 2, pp. 88-93, 2011.

[103] L. Komorowski, B. Teegen, C. Probst et al., "Autoantibodies against exocrine pancreas in Crohn's disease are directed against 
two antigens: the glycoproteins CUZD1 and GP2," Journal of Crohn's and Colitis, 2012.

[104] M. Kovacs, P. L. Lakatos, M. Papp et al., "Pancreatic autoantibodies and autoantibodies against goblet cells in pediatric patients with inflammatory bowel disease," Journal of Pediatric Gastroenterology and Nutrition, vol. 55, no. 4, pp. 429-435, 2012.

[105] D. Roggenbuck, D. Bogdanos, and K. Conrad, "Loss of tolerance to one or two major targets in Crohn's disease or just crossreactivity?" Journal of Crohn's and Colitis, 2012. 


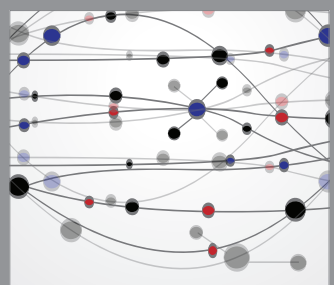

The Scientific World Journal
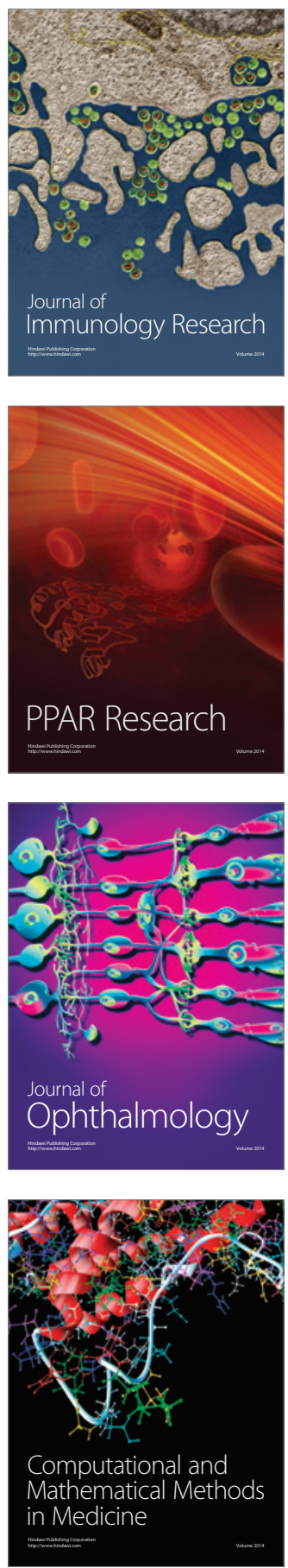

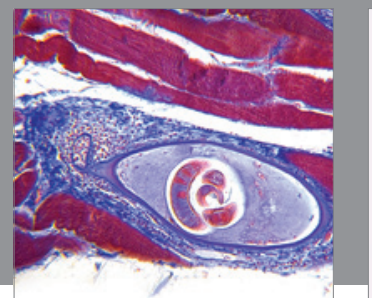

Gastroenterology

Research and Practice
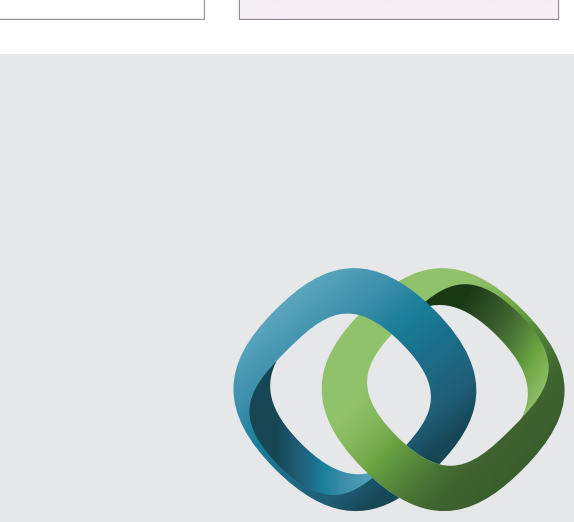

\section{Hindawi}

Submit your manuscripts at

http://www.hindawi.com
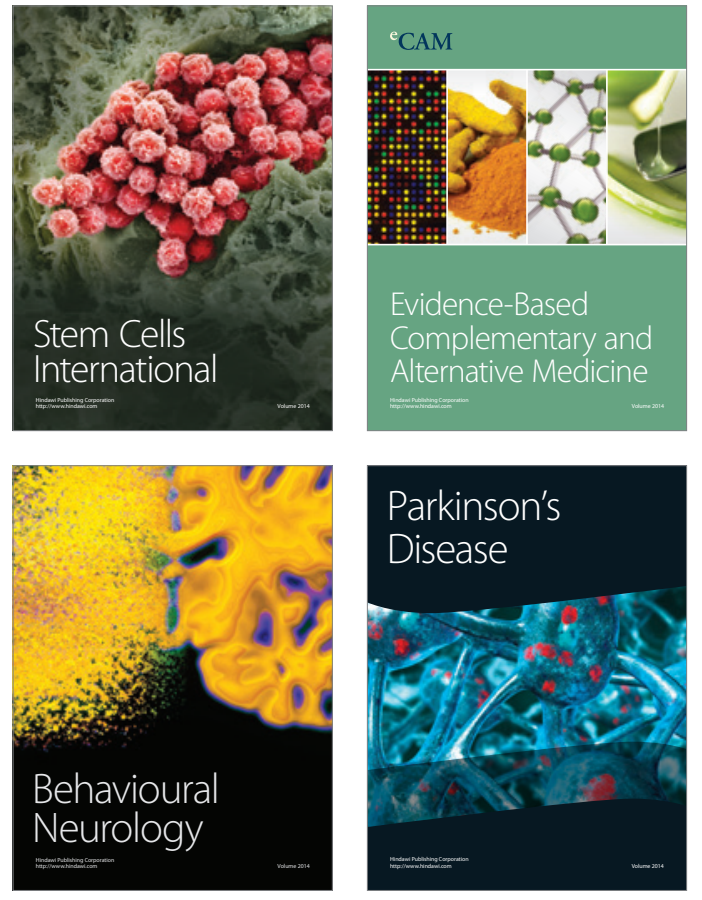
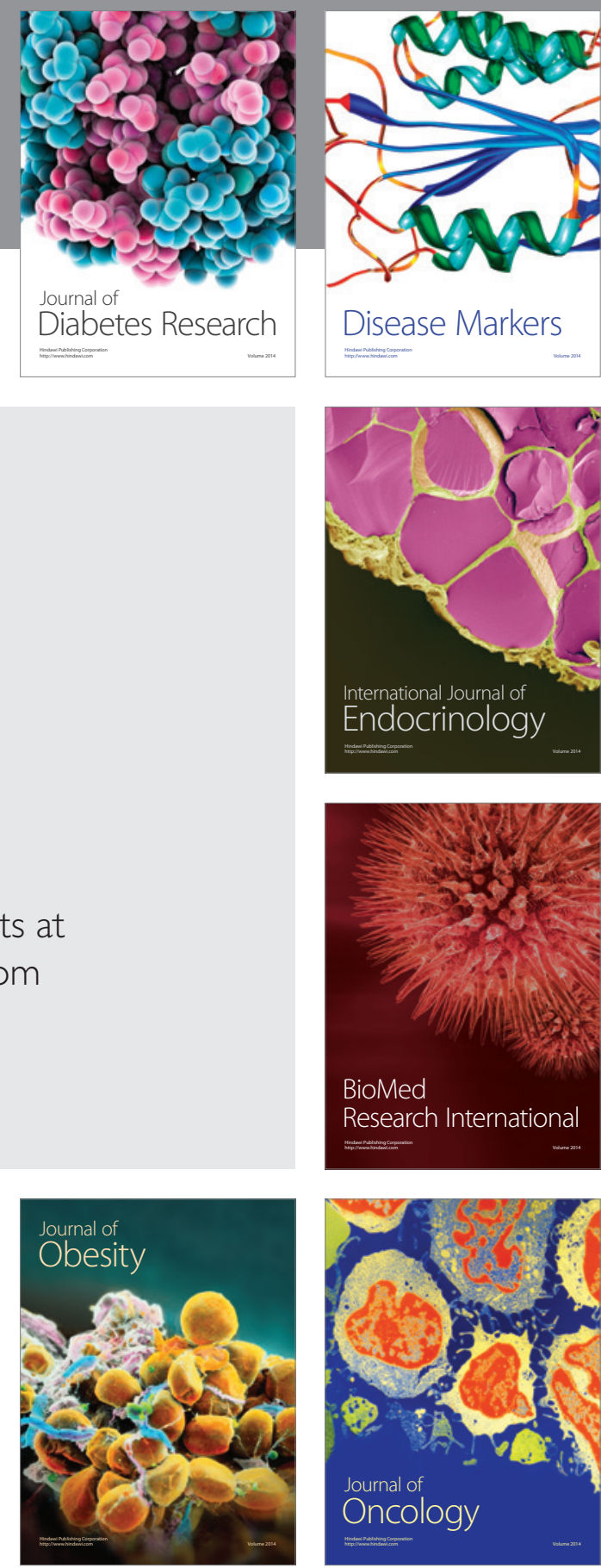

Disease Markers
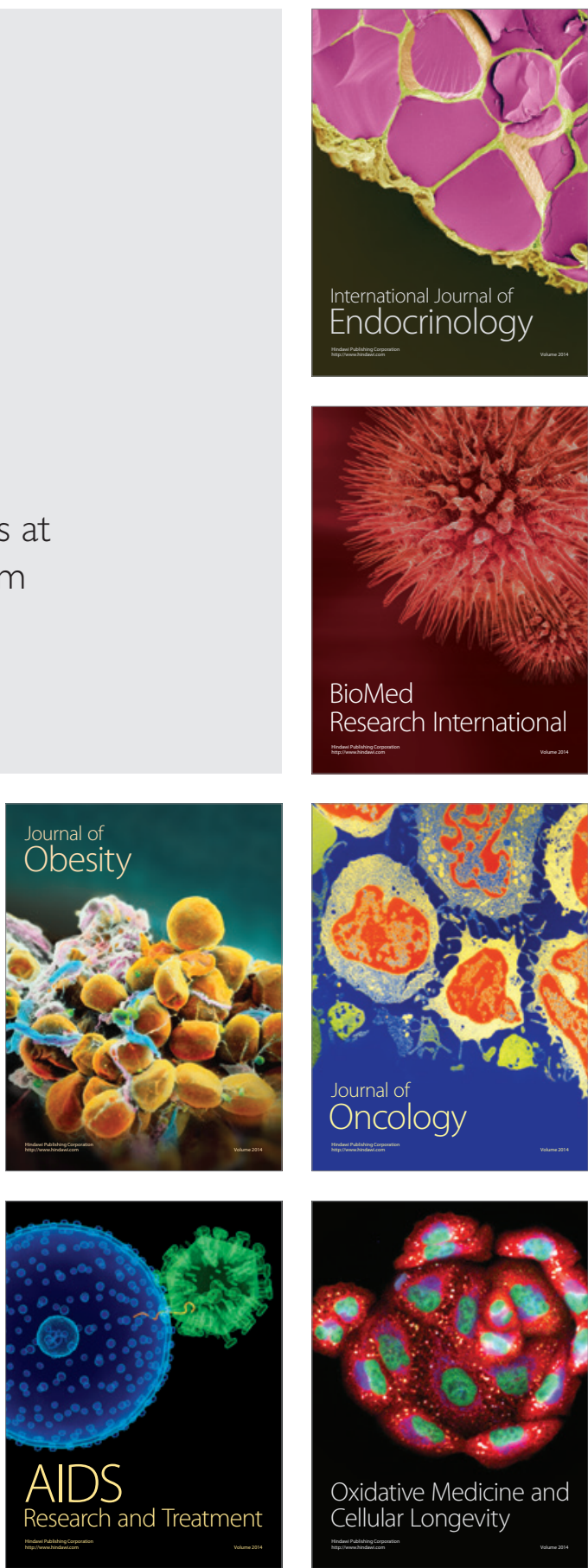